\title{
Anastomotic Leak after Colorectal Surgery: Leak Rate for Right Hemicolectomy may be Higher than Expected
}

\author{
Ozgen Isik ${ }^{\star}$, Tuncay Yilmazlar, Ersin Ozturk, Pinar Sarkut
}

Department of Surgery, Uludag University School of Medicine, Bursa, Turkey

*Corresponding author: Ozgen Isik, Uludag, University School of Medicine, Department of Surgery, Gorukle Kampusu, Bursa $16059 /$ Turkey, Tel: +90 224 295 2040; E-mail: ozgenisik@uludag.edu.tr

Received date: March 12, 2015; Accepted date: Mar 23, 2015; Published date: Mar 30, 2015

Copyright: ( 2015 Isik O, et al. This is an open-access article distributed under the terms of the Creative Commons Attribution License, which permits unrestricted use, distribution, and reproduction in any medium, provided the original author and source are credited.

\begin{abstract}
Background: Despite of the increasing knowledge and developing technology anastomotic leak (AL) is still a serious complication of colorectal surgery. The aim of this study was to evaluate the management and outcomes of $\mathrm{AL}$ after colorectal surgery.
\end{abstract}

Methods: The study included all patients diagnosed with $\mathrm{AL}$ after colorectal surgical procedures between January 2005 and December 2009. Patient demographics, diagnoses, AL management protocols, and outcomes were reviewed. Since the highest AL rate was detected for right hemicolectomy, two groups were identified based on the index surgery in order to determine the differences between the patients: right hemicolectomy and other procedures.

Results: In total, 28 of $550(5.1 \%)$ patients that underwent colorectal surgical procedures were diagnosed with $\mathrm{AL}$. There were 24 males with a median age of 61 (35-93) years. Right hemicolectomy was associated with the highest procedure-specific $\mathrm{AL}$ rate $(10.44 \%)$. In the right hemicolectomy group, patients had higher ASA scores, underwent more frequent emergency procedures, and majority of them were operated by residents comparing to the other procedures group $(p<0.05)$. Twenty-four patients required surgical intervention for $A L$, and 23 patients that underwent re-operation required a stoma; end colostomy was the most frequent procedure (50\%). AL-specific morbidity rate was $57.1 \%$ whereas the mortality rate among the patients with $\mathrm{AL}$ was $25 \%$.

Conclusion: AL after colorectal surgery is associated with high morbidity and mortality. Patients with high ASA score, emergency procedure, and inexperienced surgeon may be factors requiring extra attention in terms of high $\mathrm{AL}$ risk after right hemicolectomy comparing to the other colorectal procedures.

Keywords: Anastomotic leak; Colorectal surgery; Mortality; Morbidity

\section{Introduction}

Anastomotic leak (AL) is a serious complication of colorectal surgery and can be defined as luminal contents leaking from a surgical connection between 2 hollow viscera [1]. AL is responsible for up to $40 \%$ postoperative mortality rate, prolonged hospitalization, and an increase in the cost of healthcare due to the treatment of sepsis and the need for re-operate [2]. It is reported that in addition to an increase in the postoperative mortality rate, AL may lead poor long-term functional outcomes, increase the local recurrence rate, and decrease the 5-year cancer-specific survival rate [3-5].

The incidence of $\mathrm{AL}$ following gastrointestinal surgery varies according to the localization of anastomosis (anterior resection: 12\%-15.3\%; all resections: $4.3 \%-13 \%$ ) [6]. These variations also depend on the differences in the definition of $\mathrm{AL}$, and its clinical or radiological diagnosis. AL can be diagnosed via radiological modalities or contrast-enhanced studies; however, in some cases clinical findings alone are sufficient for determining if re-operation is necessary.

The risk factors of AL can be classified into 2 groups: patient related, and procedure related. Clinical awareness based on research that has identified age, gender, preoperative nutritional status, functional health status, blood transfusion, anastomotic tension, and emergency resection as risk factors can help surgeons avoid AL [2].

Despite colorectal subspecialty training, developments in medical technology, new operating techniques, improved intensive care resources, new broad-spectrum antibiotic drugs, utilization of complex radiological modalities, and interventional radiological support, $\mathrm{AL}$ remains the most common catastrophic complication of colorectal surgery. Herein we present the management strategies and outcomes in patients with AL treated at a tertiary, high-volume referral center in Turkey.

\section{Material and Methods}

In total, 550 patients that underwent colorectal surgical procedures between January 2005 and December 2009 were retrospectively reviewed. Colorectal procedures with anastomosis, without stoma (except Hartmann's procedure) and fecal diversion were included. Patients younger than 18, and patients with American Society of Anesthesiologists (ASA) score 5 or 6 were excluded.

Patients who were diagnosed with AL within 30 days after index surgery were identified. Radiologically and clinically diagnosed AL's were both included. Patient demographics, characteristics, primary 
Citation: Isik O, Yilmazlar Y, Ozturk E, Sarkut P (2015) Anastomotic Leak after Colorectal Surgery: Leak Rate for Right Hemicolectomy may be Higher than Expected. J Integr Oncol 4: 130. doi:10.4172/2329-6771.1000130

Page 2 of 4

diagnoses, index surgical procedures, AL management protocols, and morbidity and mortality rates were extracted from the patients' medical records. AL was defined as luminal contents leaking from the surgical anastomosis between 2 hollow viscera.

We detected a higher AL rate for right hemicolectomy procedure comparing to the reported rates in the literature. In order to determine the factors increasing the $\mathrm{AL}$ rate after right hemicolectomy, we compared them with the other AL patients (right hemicolectomy versus other procedures). Categorical variables were reported as frequency (\%) and quantitative variables were reported as mean \pm standard deviation except otherwise noted. Categorical variables were compared by using the Fisher exact or Chi-square test, and quantitative and ordinal variables by using the Wilcoxon rank sum test. A p value of $<0.05$ was considered statistically significant. All statistical analyses were performed using JMP version 10.0 (SAS Institute Inc, Cary, North Carolina).

\section{Results}

In total, 28 of 550 (5.1\%) patients that underwent colorectal surgical procedures were diagnosed with AL. There were 24 males and 4 females, with a median age of 61 (35- 93) years. In all, 12 patients were current smokers. Eleven patients had comorbidities: hypertension $(n=4)$, cardiac arrhythmia $(n=3)$, coronary artery disease $(n=2), D M$ $(\mathrm{n}=2)$, asthma $(\mathrm{n}=1)$, toxic multinodular goiter $(\mathrm{n}=1)$, and arthritis $(n=1)$. Primary indications for surgery are shown in (Figure 1).

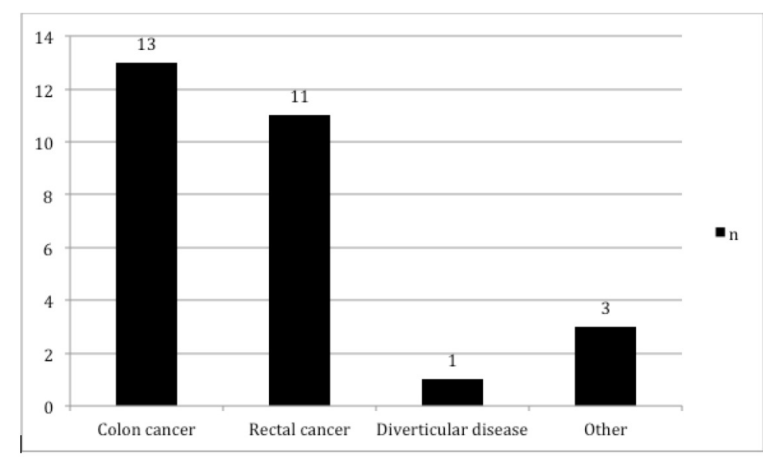

Figure 1: Primary diagnoses in the patients with $\mathrm{AL}$
Three out of 28 patients were performed an emergency procedure. Majority of the procedures were performed by attending surgeons (75\%). Median operating time was $120 \mathrm{~min}$ (range: $60-180 \mathrm{~min}$ ) and 11 patients had a hand-sewn anastomosis. A summary of the surgical procedures performed and procedure-specific leakage rates are shown in Table 1. Right hemicolectomy was associated with the highest procedure-specific AL rate (10. 44\%). Results of the analysis that compares AL's after right hemicolectomy with AL's after other procedures are shown in Table 2.

\begin{tabular}{|l|l|l|l|}
\hline Procedure & $\mathbf{n}$ & Leakage (n) & $\begin{array}{l}\text { Leakage rate } \\
(\%)\end{array}$ \\
\hline LAR $^{\beta}$ & 111 & 8 & 7.2 \\
\hline Ileostomy reversal & 84 & 4 & 4.76 \\
\hline AR $^{\alpha}$ & 72 & 3 & 4.16 \\
\hline Right Hemicolectomy & 67 & 7 & 10.44 \\
\hline Hartmann's procedure & 55 & - & - \\
\hline Hartmann's reversal & 52 & 1 & 1.92 \\
\hline Left hemicolectomy & 34 & 3 & 8.82 \\
\hline Total colectomy + IRA ${ }^{\theta}$ & 27 & 2 & 7.4 \\
\hline Others & 48 & - & - \\
\hline Total & 550 & 28 & 5.1 \\
\hline$\beta:$ low anterior resection; $\alpha:$ anterior resection; $\theta:$ ileorectal anastomosis \\
\hline
\end{tabular}

Table 1: The frequency of colorectal surgical procedures and procedure-specific leakage rates

Median AL diagnosis time was 7.5 (3-20) days. In all, 22 (78.5\%) patients had subsequent fever, tachycardia, and peritoneal irritation, which were the most prominent clinical findings that cast doubt on the diagnosis of AL. Among the patients, 8 (28.57\%) with enteric fluid drainage from the incision or drain re-operated without requiring any other diagnostic work up, whereas 15 patients underwent computerized tomography (CT) and 1 underwent colonoscopy (presence of hematochezia) for diagnostic purposes. CT was false negative in 3 patients. The diagnostic accuracy of CT was $80 \%$. Leukocytosis and elevated urea and creatinine levels were supportive laboratory findings for the diagnosis of $\mathrm{AL}$.

\begin{tabular}{|c|c|c|c|c|}
\hline & & Right Hemicolectomy $(n=7)$ & Other Procedures $(n=21)$ & $P$ value \\
\hline Age (years) & & $62(44-74)$ & $61(35-93)$ & 0.94 \\
\hline Female gender & & $14.30 \%$ & $14.30 \%$ & $>0.99$ \\
\hline \multirow[t]{5}{*}{ ASA§ score } & & & & 0.004 \\
\hline & 1 & - & $61.90 \%$ & \\
\hline & II & - & $14.30 \%$ & \\
\hline & III & $57.10 \%$ & $19 \%$ & \\
\hline & IV & $42.90 \%$ & $4.80 \%$ & \\
\hline
\end{tabular}




\begin{tabular}{|l|l|l|l|l|}
\hline Primary indication for surgery (malignancy) & $85.70 \%$ & $85.70 \%$ & $>0.99$ \\
\hline Emergency procedure & $42.90 \%$ & - & 0.01 \\
\hline Surgeon (attending) & $42.90 \%$ & $85.70 \%$ & 0.04 \\
\hline Operating time (min) & $90(90-180)$ & $120(60-180)$ & 0.25 \\
\hline Anastomotic technique (handsewn) & $71.40 \%$ & $28.60 \%$ & 0.07 \\
\hline Postoperative time of AL đI diagnosis (days) & $6(4-9)$ & $8(3-20)$ & 0.25 \\
\hline Length of hospital stay (days) & $18(7-90)$ & $29(9-72)$ & 0.23 \\
\hline Morbidity & $71.40 \%$ & $61.90 \%$ & 0.64 \\
\hline Mortality & $57.10 \%$ & $14.30 \%$ & 0.04 \\
\hline §: American Society of Anesthesiologists; T: Anastomotic leak & & \\
\hline
\end{tabular}

Table 2: Comparison of AL patients after right hemicolectomy and other procedures

In total, 24 patients required surgical intervention, whereas 4 patients did well with percutaneous drainage and antibiotics. In total, 23 patients that underwent re-operation required a stoma; end colostomy was the most frequent procedure $(n=12)$. In all, 4 patients underwent end ileostomy, 4 double barrel colostomy, 2 ileostomy with mucous fistula, 1 loop ileostomy, and 1 surgical drainage. Among the re-operated patients, 8 required multiple surgical interventions.

The overall morbidity rate was $7.8 \%$ (43 of 550), whereas the ALspecific morbidity rate was $57.1 \%$ (16 of 28$)$. The most common morbidity among the patients with AL was surgical site infection (SSI) (7 of 28). Pneumonia $(n=4)$, intraabdominal abscess $(n=4)$, cardiac arrhythmia $(n=2)$, hematochezia $(n=1)$, acute renal failure $(n=1)$, and necrotizing fasciitis $(\mathrm{n}=1)$ were other morbidities noted in the patients. The overall mortality rate was $4.18 \%$ ( 23 of 550 patients), whereas the mortality rate among the patients with $\mathrm{AL}$ was $25 \%$ (7 of 28 ). The most common cause of death was post AL sepsis. Only 1 patient died due to myocardial infarct in the early postoperative period.

Median duration of hospitalization among the patients with AL was 26 (7-90) days. In all, 18 patients were followed-up in the intensive care unit (ICU) for a median 2 (0-22) days. The mortality rate among the patients that required ICU follow-up was $38.88 \%$ ( 7 of 18), whereas none of the patients followed-up in the regular nursing unit died.

\section{Discussion}

Despite an increase in our understanding of $\mathrm{AL}$ and advancements in surgical techniques, AL remains a serious problem than can even occur in patients without any risk factors. This study showed that AL after colorectal surgery is associated with high morbidity and mortality. Although right hemicolectomy is generally considered as a safe procedure in terms of $\mathrm{AL}$ occurrence, our results suggest that surgeons should never underestimate the risk of $\mathrm{AL}$ even after right hemicolectomy.

The incidence of $\mathrm{AL}$ after colorectal surgery was reported as $1 \%-30 \%$ in several prospective, retrospective, and population-based cohort studies [7]. In the present study, AL rate was $5.1 \%$. Variability in the incidence of $\mathrm{AL}$ is related to the lack of consensus concerning the definition and diagnosis of AL. However, it is still a serious complication of colorectal surgery that reduces cancer-specific survival and causes $25 \%$ of postoperative mortality [3-5].

The most common radiological diagnostic work up for AL in the present study was $\mathrm{CT}$, which yielded a preoperative diagnostic accuracy of $80 \%$. It was reported earlier that rectal contrast-enhanced $\mathrm{CT}$ is better than contrast enema for diagnosing post-colectomy $\mathrm{AL}$, intraabdominal abscess, and pelvic abscess $[6,8]$. The most important CT finding associated with AL is a perianastomotic loculated fluid collection containing air [9]. In a recently published study, the preoperative diagnostic accuracy of CT was $68 \%$ in patients with suspected AL following colorectal resection [10]. We recommend CT as the first-line diagnostic tool for patients with suspected AL.

In the present study $10.44 \%$ of patients that underwent right hemicolectomy had AL; an intriguing finding that requires additional investigation. Our results suggest that patients with $\mathrm{AL}$ after right hemicolectomy had higher ASA scores; more frequently underwent emergency procedure and more frequently operated by residents comparing to the patients with $\mathrm{AL}$ after other procedures. Right hemicolectomy is widely considered an educational procedure for residents. Since it has a potent blood supply, and is usually tensionfree, an ileocolic anastomosis encourages surgeons that it is safer than other colon or rectal anastomoses which can lead to a delay in the diagnosis of AL. However, it was reported that the mortality rate due to AL after right hemicolectomy was higher than other procedures [3]. Advanced age in patients with right colon localized diseases, and comorbidities related to advanced age in such patients might be associated with an increase in AL-associated mortality. Nevertheless, surgeons' experience, and emergency procedures are also important factors influencing AL risk after right hemicolectomy procedure.

Patients with AL require aggressive intravenous fluid replacement and broad-spectrum antibiotic administration. Treatment must be individualized, according to patient need. Treatment options include observation and bowel rest, percutaneous drainage, colonic stent placement, and surgical revision, diversion, or drainage. In the present study, 4 patients were treated with non-surgical methods, whereas 24 underwent re-surgery; the most common procedure performed to treat $\mathrm{AL}$ was end colostomy. The primary aim of re-operation is to control the leak and provide adequate drainage. End colostomy, or proximal diversion may be preferred. It was reported that stoma 
reversal failed in $56 \%$ of patients with end stoma, and in $31 \%$ of patients with proximal diversion during 31.7 months of follow-up [11]. Moreover, it was reported that stoma reversal rates were higher if the leak was successfully controlled during primary surgery for AL. Repair or revision of anastomosis alone is not recommended because of the high mortality rate associated with recurrent AL [11]. Endostent use and transanal endoscopic vacuum devices may be alternative treatment options in selected patients with $\mathrm{AL}[12,13]$.

Infectious complications, such as septicemia, pelvic and intraabdominal abscess, and infected pelvic hematoma, are common in patients with AL. In addition, urinary, pulmonary, and prolonged ileus complications are not rare [14]. The overall morbidity rate was $57.1 \%$ in the present study; the most common morbidity was deep SSI, followed by intraabdominal abscess, pneumonia, cardiac arrhythmias, gastrointestinal bleeding, acute renal failure, and necrotizing fasciitis of the abdominal wall. Alves et al. similarly reported that pneumonia, surgical site infections, and urinary infections were the most common causes of morbidity. They also reported that patients with AL are more prone to develop morbidity than those without AL. They suggested that $\mathrm{AL}$ is associated with prolonged hospitalization, because of the higher morbidity rate [15]. Although intraabdominal abscess formation and AL have a negative effect on recurrence and 5-year survival in patients with rectal cancer, they do not have such an effect in patients with colon cancer [16].

It was reported that the mortality rate associated with AL is $2 \%-39 \%$ [15]; in the present study the mortality was $25 \%$. A study that compared gastrointestinal anastomoses reported that $\mathrm{AL}$ was common following total gastrectomy (4.8\%) and esophagogastrectomy (4.4\%), whereas the highest mortality rate was associated with partial gastrectomy (75\%), followed by esophagogastrectomy (50\%). The same study reported that the AL rate associated with partial colectomy and subtotal colectomy were $1.7 \%$ and $3 \%$, respectively, and that the associated mortality rate were $4.8 \%$ and $14.3 \%$, respectively [17]. These findings further indicate that $\mathrm{AL}$ is more common after upper gastrointestinal surgical procedures than colorectal procedures. Although numerous studies have investigated AL in colorectal surgery during the previous decade, the mortality rate has not decreased [15]. In the present study, the incidence of $\mathrm{AL}$ and associated mortality rate were comparable to those previously reported.

The major limitation of the present study is its retrospective design. On the other hand, patients of advanced age with comorbidities, and advanced-stage cancer requiring neoadjuvant chemotherapy are commonly referred to our hospital; thus, it is a tertiary referral center for its region, which might have caused selection bias. Nonetheless, as the study was conducted at just one colorectal surgery department and high-volume colorectal surgeons performed or supervised all the procedures, this research has clinical significance.

In conclusion, $\mathrm{AL}$ after colorectal surgery remains a serious problem despite advances in medical technology and surgeon experience. Right hemicolectomy may be associated with a higher AL rate in patients with high ASA score, that underwent emergency procedure, and that operated by an inexperienced surgeon. Early diagnosis, effective drainage, and leakage control are essential for the treatment of AL. Surgeons should not avoid end-stoma formation for achieving effective disease control. Despite the best surgical techniques and medical treatment, mortality cannot be prevented in all patients because of variation in the human immune response to sepsis.

\section{References}

1. Leichtle SW, Mouawad NJ, Welch KB, Lampman RM, Cleary RK (2012) Risk factors for anastomotic leakage after colectomy. Dis Colon Rectum 55: 569-575.

2. Boccola MA, Buettner PG, Rozen WM, Siu SK, Stevenson AR, et al. (2011) Risk factors and outcomes for anastomotic leakage in colorectal surgery: a single-institution analysis of 1576 patients. World J Surg 35: 186-195.

3. Thornton M, Joshi H, Vimalachandran C, Heath R, Carter P, et al. (2011) Management and outcome of colorectal anastomotic leaks. Int J Colorectal Dis 26: 313-320.

4. Katoh H, Yamashita K, Wang G, Sato T, Nakamura T, et al. (2011) Anastomotic leakage contributes to the risk for systemic recurrence in stage II colorectal cancer. J Gastrointest Surg 15: 120-129.

5. Mirnezami A, Mirnezami R, Chandrakumaran K, Sasapu K, Sagar P, et al. (2011) Increased local recurrence and reduced survival from colorectal cancer following anastomotic leak: systematic review and meta-analysis. Ann Surg 253: 890-899.

6. Khan A, Wheeler J, Cunningham C, George B, Kettlewell M, et al. (2008) The management and outcome of anastomotic leaks in colorectal surgery. Colorectal Dis 10: 587-592.

7. Kingham TP, Pachter HL (2009) Colonic anastomotic leak: risk factors, diagnosis, and treatment. J Am Coll Surg 208: 269-278.

8. Bellows C, Webber L, Albo D, Awad S, Berger D (2009) Early predictors of anastomotic leaks after colectomy. Tech Coloproctol 13: 41-47.

9. Power N, Atri M, Ryan S, Haddad R, Smith A (2007) CT assessment of anastomotic bowel leak. Clin Radiol 62: 37-42.

10. Kornmann VN, Treskes N, Hoonhout LH, Bollen TL, van Ramshorst B, et al. (2013) Systematic review on the value of CT scanning in the diagnosis of anastomotic leakage after colorectal surgery. Int J Colorectal Dis 28: 437-445.

11. Francone TD, Saleem A, Read TA, Roberts PL, Marcello PW, et al. (2010) Ultimate fate of the leaking intestinal anastomosis: does leak mean permanent stoma? J Gastrointest Surg 14: 987-992.

12. Arezzo A, Miegge A, Garbarini A, Morino M (2010) Endoluminal vacuum therapy for anastomotic leaks after rectal surgery. Tech Coloproctol 14: 279-281.

13. Abbas MA (2009) Endoscopic management of acute colorectal anastomotic complications with temporary stent. JSLS 13: 420-424.

14. Tytherleigh MG, Bokey L, Chapuis PH, Dent OF (2007) Is a minor clinical anastomotic leak clinically significant after resection of colorectal cancer? J Am Coll Surg 205: 648-653.

15. Alves A, Panis Y, Pocard M, Regimbeau JM, Valleur P (1999) Management of anastomotic leakage after nondiverted large bowel resection. J Am Coll Surg 189: 554-559.

16. Eberhardt JM, Kiran RP, Lavery IC (2009) The impact of anastomotic leak and intra-abdominal abscess on cancer-related outcomes after resection for colorectal cancer: a case control study. Dis Colon Rectum 52: 380-386.

17. Pickleman J, Watson W, Cunningham J, Fisher SG, Gamelli R (1999) The failed gastrointestinal anastomosis: an inevitable catastrophe? J Am Coll Surg 188: 473-482. 